\title{
Validity of the Doppler velocimeter in examination of vertebral artery blood flow and its use in pre-manipulative screening of the neck.
}

Lucy C. Thomas ${ }^{1}$ DipPhys, GradDipAppSc(ManipPhty), MMedSc (Physiotherapy)

Darren A. Rivett ${ }^{2}$ BAppSc(Phty), MAppSc(ManipPhty), PhD

Philip S. Bolton ${ }^{3,4}$ BSc, DC, PhD

Affiliations

1) Lecturer, Discipline of Physiotherapy, School of Health Sciences

2) Associate Professor, Discipline of Physiotherapy, School of Health Sciences

3) Associate Professor, Discipline of Human Physiology, School of Biomedical Sciences

Faculty of Health

The University of Newcastle

NSW, Australia

4) Member, Hunter Medical Research Institute, New Lambton, NSW, Australia

Address for correspondence:

Lucy Thomas

Discipline of Physiotherapy

School of Health Sciences

Faculty of Health

The University of Newcastle

University Drive

Callaghan 2308

NSW, Australia

Telephone: +61249218680 Fax: +61249217053

e-mail: Lucy.Thomas@newcastle.edu.au 
Validity of the Doppler velocimeter in examination of vertebral artery blood flow and its use in pre-manipulative screening of the neck. 
Abstract

Pre-existing compromise of one or both vertebral arteries is considered a contraindication to neck manipulation. Current pre-manipulative screening tests may not adequately identify individuals with such compromise. It has been proposed that using a continuous wave ultrasound device (Doppler velocimeter) may assist in identifying patients presenting with flow abnormalities. The aim of this study was to determine the validity and reliability of the use of a velocimeter in detecting altered vertebral artery blood flow. Blood flow in the atlanto-axial segment of seated healthy adult volunteers $(n=60)$ was examined in the neutral and end-range contralateral rotation positions. Duplex ultrasound scans were performed $(n=58)$ and identified seventeen volunteers (29.3\%) with abnormal flow according to pre-determined criteria. Three trained physiotherapists blinded to the duplex examination results used a velocimeter to examine the vertebral arteries of the volunteers. The specificity of the velocimeter examination to detect abnormal flow identified by the duplex examination was fair to good (range $0.78-0.88$ ). However, its sensitivity was poor (range $0.25-0.38$ ) and the inter-examiner reliability was poor ( $\mathrm{k}$ ranged from $0.15-0.26)$. This study suggests that the velocimeter may be neither a valid or reliable tool for the detection of abnormal blood flow in the vertebral arteries.

Keywords

Vertebral artery, cervical manipulation, cervical vertebrae, ultrasonic diagnosis 


\section{$\underline{\text { Introduction }}$}

Stroke is a rare but devastating consequence of cervical spine manipulation (Thiel et al. 2007; Cassidy et al. 2008), and is thought to be associated with emboli or injury of the vertebral artery (VA) (Frisoni \& Anzola 1991; Lee et al. 1995; Cassidy et al. 2008). In a recent review (Thomas et al. 2008) we critically considered the validity of clinical tests intended to identify people at risk of vertebrobasilar insufficiency associated with cervical spine manipulation. In addition, we considered the merit of using a portable continuous wave Doppler device, known as a velocimeter, to examine the integrity of VA blood flow prior to neck manipulation (Thomas et al. 2008).

The criterion standard for examining extracranial arterial patency is magnetic resonance angiography (MRA) (Zwiebel 2000), however this is a specialized investigation not readily available to manual therapy practitioners. Duplex ultrasound has also been used to examine VA blood flow and while there can be some difficulties with accuracy associated with its use, it would appear to offer the most direct and least invasive method of measuring VA blood flow.

Haynes (2002a) and Rivett (2001) have each proposed the use of a handheld Doppler velocimeter as a simple screening tool to provide an objective means for clinicians to assess VA blood flow. Haynes has reported that the velocimeter has an inter-rater reliability of 0.78 and a sensitivity and specificity of $100 \%$ for identifying 'persistent' or 'major reduction' in VA blood flow when compared to findings from duplex ultrasound (Haynes 2000; Haynes et al. 2000). We have undertaken and report here, a further study of the sensitivity and specificity of the velocimeter to identify altered blood flow in the VA. In contrast to Haynes (2000), all participants in our study underwent both duplex ultrasound examination and velocimeter examination of the VA using a double blind study format.

The specific aims of this study were to determine whether physiotherapists, trained in the use of the Doppler velocimeter, are able to reliably detect VA blood flow 'abnormalities' in the atlanto- 
axial segment of the VA and to determine the sensitivity and specificity of this Doppler velocimeter examination when compared to duplex ultrasound examination.

\section{Method}

The protocol used in this study was approved by the University of Newcastle Human Research Ethics Committee and the Hunter Area Research Ethics Committee. The study was performed in accordance with the 2007 National Statement on ethical conduct in research involving humans (NHMRC 2007).

The study was undertaken in two stages. Firstly, participants were examined with duplex ultrasound by a qualified ultrasonographer, to determine their VA blood flow status. Secondly, participants were examined by three physiotherapists using a Doppler velocimeter, according to a protocol described by Haynes (2000).

\section{Recruitment}

Healthy adult volunteers aged between 18 to 80 years were recruited via advertisement. Exclusion criteria were history of cervical spine instability, inflammatory disease or uncontrolled cardiovascular disease which would normally contraindicate cervical manipulation. Eligible volunteers became participants in the study once they gave their informed consent to take part.

Three local physiotherapists were recruited to perform the velocimeter examinations. Inclusion criteria were registration as a physiotherapist in New South Wales, at least five years clinical experience in the field of manual therapy and familiarity with the current Australian Physiotherapy Association Clinical Guidelines for Pre-manipulative Procedures for the Cervical Spine (Magarey et al. 2000).

\section{Protocols}

Each participant underwent examination of their left and right VA with a Phillips HDI 3000 Duplex ultrasound scanner (ATL Philips, Bothel, USA) with colour flow imaging. A $38 \mathrm{~mm}$ broadband 
linear transducer with a frequency of between 5-12 $\mathrm{MHz}$ (mean $6 \mathrm{MHz}$ ) was used by a qualified ultrasonographer experienced in vascular imaging, who had additional training to the specific requirements of the study.

To facilitate stabilisation of blood pressure (BP) and heart rate (HR), each participant was asked to rest in a relaxed seated position for ten minutes prior to duplex or velocimeter examination. These parameters were measured before and after scanning using a digital BP monitor with an accuracy of $\pm 3 \mathrm{mmHg}$ for BP and $\pm 5 \%$ for HR (Nissei digital BP monitor, model DS-105E, Nihon Seimitsu Co. Ltd, Japan). Any individual who had high BP (systolic $\geq 180 \mathrm{mmHg}$ ) as defined by the Australian Heart Foundation (Heart Foundation 2004) was excluded from the study because this is a contraindication to cervical manipulation (George et al. 1981) and because their BP could vary due to their condition and confound the results of the ultrasound examinations.

A cervical range of motion device (CROM) (Performance Attainment Associates, Minnesota, USA) was used to measure cervical range of rotation during the ultrasound procedures. This device has been demonstrated to have good (ICC 2:1 >0.80) intra-therapist reliability in measurement studies of cervical range of movement (Capuano-Pucci et al. 1991; Youdas et al. 1991; Rheault et al. 1992).

\section{Duplex examination}

The participant was seated beside the scanner. The ultrasonographer applied conducting gel to the broadband transducer and placed the probe in the sub-occipital region just posterior to the mastoid process. Using real-time imaging and colour flow mapping, blood flow in the VA was identified between the atlas and axis, where it exits from the axial transverse foramen. After thirty seconds in the neutral position, the Doppler waveform trace was recorded, with values for peak systolic (PS), end diastolic (ED) and time averaged (TAV) velocities. Each velocity was averaged over five cardiac cycles. The participant was then asked to turn their neck slowly to the 
contralateral side while the ultrasonographer followed with the transducer, maintaining visual contact with blood flow on the real-time image screen. Once maximum rotation was achieved, adjustments to the cursor for midstream sampling and angle correction ( $\leq 60$ degrees) were made by the ultrasonographer for the new position to ensure optimum signal. This position was maintained for a minimum of thirty seconds before the Doppler waveform trace and velocity values were again recorded. The participant's maximum range of neck rotation was simultaneously recorded by another examiner using the CROM device. The participant then returned their neck to the neutral position and rested for 30 seconds. The procedure was then repeated for the opposite VA. Throughout the examination procedure the participant was carefully monitored for any symptoms of vascular compromise (such as dizziness, paraesthesia) or pain. The entire ultrasound examination procedure was repeated for each VA.

Following the duplex examination, participants were invited to attend a local physiotherapy clinic for the velocimeter examination.

\section{Velocimeter examination}

The velocimeter examination was performed by each of three physiotherapists using a Huntleigh Super Dopplex II velocimeter (Huntleigh Diagnostics, Perth, Western Australia). This bidirectional continuous wave Doppler velocimeter was used with a $4 \mathrm{MHz}$ handheld probe, designed specifically for detecting blood flow in deep lying vessels.

The examiners first undertook a three hour training workshop during which they were briefed on the experimental protocol for the velocimeter examination of the VA and recording of findings. Each was provided with opportunity to practice the examination technique under the supervision of one of the investigators who had received training from an experienced user of the velocimeter. During the workshop, audio recordings of abnormal VA blood flow were used to train the physiotherapists. At the conclusion of the workshop the examiners were each loaned a velocimeter and asked to use it three to four times per week over a two month period on different subjects. This was done to emulate the typical frequency of use of pre-manipulative tests by 
physiotherapists (Magarey et al. 2004). At the conclusion of the training period the same investigator assessed the examiners by means of an oral and practical test to ensure minimum standards of competency.

Participants underwent velocimeter examination by each of the trained examiners in random order. One examiner did not examine one participant because the participant was known to the examiner. Participants were first seated and rested for ten minutes to facilitate stabilisation of BP and HR which were measured at the conclusion of this acclimatisation period. The CROM was then attached to the participant. Each participant had both VAs examined at the atlanto-axial segment according to the protocol described by Haynes (2000). In brief, the examiner directed the velocimeter probe to the sub-occipital level posterior to the mastoid process and angled to a point midway along an imaginary arc between the eyes of the participant. The examiner sought the characteristic low resistance Doppler auditory signal of the VA which is described as a 'soft whiplash' or two beat sound. The probe position was maintained for thirty seconds to confirm the presence and stability of the blood flow signal. The participant was then instructed to turn their head and neck as far as possible to the contralateral side while the probe was manoeuvred to ensure the VA signal remained present. Maximum range of cervical rotation was recorded with the CROM. The examiner then recorded their velocimeter findings categorising blood flow as being either 'normal' or 'abnormal' on the basis of the auditory Doppler signal. Blood flow was categorised as abnormal if any of the criteria previously described by Haynes (2000) (see Table 1) were met.

Table 1 about here

This procedure was repeated for the opposite VA. If an examiner failed to detect VA flow at the sub-occipital portal or lost the signal during rotation, the procedure was repeated at the axial level to confirm the lack of flow or to determine if flow could be detected at this axial (lower) level. In this position the probe was directed at the transverse process of the axis and angled medially and slightly superiorly in order to sample the blood flow end-on (i.e. moving towards the probe) where the artery forms a convex loop infero-laterally emerging from the transverse foramen, as 
described by Haynes (2002a). The examiner was permitted to re-search for flow if they were unable to follow the flow round during rotation.

At the conclusion of the velocimeter examination BP and HR were measured again.

Data analysis

All data was tabulated and a descriptive statistical analysis was performed on the group data to determine the range, mean and standard deviation values of the study sample.

In order to determine the reliability of the duplex examination, repeat PS, ED and TAV velocity scores were assessed using intra-class correlation coefficients (ICC 2, 1) with 95\% confidence intervals (Shrout \& Fleiss 1979).

The repeat recordings of PS, ED and TAV flow values in each head position were averaged. Using these averaged values, the difference in blood flow velocity (PS, ED, TAV) between the two head positions was determined by calculating the difference in velocity between the two positions for each individual.

Participants were categorised into those with 'normal' and 'abnormal' VA blood flow based on the results of the duplex examination and categorised as 'abnormal' if any of the criteria listed in Table 2 were met. Criteria were derived from the work of Freed et al (1998) and Zwiebel (2000).

Table 2 about here

Participants were categorised into those with 'normal' or 'abnormal' VA flow based on the velocimeter examination using the criteria listed in Table 1.

The results of the duplex ultrasound and the velocimeter examination for each of the three examiners were compared using a $2 \times 2$ contingency table. Sensitivity, specificity and likelihood 
ratios were calculated for each examiner. Cohen's kappa statistic was used to evaluate the level of agreement between each examiner's findings (Fleiss 1971; Fleiss 1986).

Statistical analysis was undertaken using SPSS 11.0 statistical package for Windows (SPSS Inc., Chicago, Illinois, USA).

\section{$\underline{\text { Results }}$}

Nineteen males and forty-one females entered the study, with an age range of 21-71 years and mean of 45.5 years $( \pm 15.5)$. No participants complained of neck pain or reported any symptoms of vertebrobasilar insufficiency entering the study, and all remained asymptomatic throughout both examinations.

The mean systolic and diastolic BP for participants was $126.53 \pm 16.81$ and $82.88 \pm 13.54 \mathrm{mmHg}$, respectively. No participants demonstrated changes in BP during the study period which would have excluded them from the study. The mean HR was $78.46 \pm 16.72$ beats per minute (bpm).

Cervical rotation during scanning ranged from $69.19 \pm 10.67$ degrees ( $R$ rotation, duplex) to 71.84 \pm 9.34 degrees (R rotation, velocimeter) and from $70.71 \pm 10.74$ degrees ( $L$ rotation, duplex) to $70.61 \pm 10.81$ degrees ( $L$ rotation, velocimeter). There was no significant difference in range of motion between the duplex or velocimeter examinations.

\section{Duplex examination}

Fifty-eight participants were successfully scanned with duplex ultrasound. Two participants were excluded due to ill-defined Doppler traces. Both excluded participants had short necks with welldeveloped musculature. This may have caused attenuation of the reflected Doppler signal.

The ICCs of the duplex ultrasonographic measurement of PS and ED ranged from 0.71 to 0.85 (see Table 3), suggesting good repeatability (Fleiss 1986) of the procedure. 
Table 3 about here

Of the 58 participants scanned with duplex ultrasound, 17 individuals (29.3\%) involving 27 VAs (see Table 4) had blood flow considered to be abnormal according to the pre-determined criteria. Figure 1 shows typical Doppler traces from two participants in the study.

Figures 1 and 2 about here

Figure 2 shows the distribution of percent change in PS velocity for all VAs. Five participants (six arteries) had PS changes of $>50 \%$. No participants demonstrated absence of PS flow in neutral or complete cessation of flow on contralateral rotation.

Twelve participants had ED findings considered to be aberrant based on the criteria in Table 2 . Table 4 shows the type of abnormal blood flow demonstrated. Abnormalities were equally distributed between sides.

Table 4 about here

Velocimeter examination

Fifty-seven participants completed the velocimeter examination. One participant was lost to follow up due to re-location. Table 5 presents a comparison of the individual results from the duplex examination and velocimeter examination findings obtained by each of the physiotherapist examiners. Table 6 summarises the sensitivity, specificity and likelihood ratios of the velocimeter examination compared with the duplex.

Tables 5 and 6 about here 
The specificity of the velocimeter examination was between 0.78 and 0.88 . This can be considered to be fair to good (Fleiss 1986). In contrast, the sensitivity of the velocimeter examination (range $0.25-0.38$ ) was poor (Fleiss 1986). Examiner 1 had an appreciably higher positive likelihood ratio.

The kappa scores ranged from $0.15-0.26$ ( $\mathrm{k}$ was 0.15 for examiners 1 and 2; 0.18 for examiners 1 and 3; and 0.26 for examiners 2 and 3). These values are below 0.40 , suggesting poor agreement between examiners (Fleiss 1986; Bogduk 1999).

\section{Discussion}

The results of the present study suggest that trained clinicians cannot reliably detect VA blood flow abnormalities in the atlanto-axial region using a Doppler velocimeter. Furthermore, Doppler velocimeter examination is not a valid method of assessing VA blood flow for the types of aberrant blood flow identified in this study.

We found the use of the velocimeter by the clinicians to have poor sensitivity $(0.25-0.38)$ in detecting aberrant VA blood flow demonstrated by duplex. This suggests that use of the velocimeter, at least in this study, would result in a high proportion of false negative findings. The specificity of the velocimeter examination was fair to good $(0.78-0.88)$, suggesting the examination would yield few false positive results. Nonetheless, the likelihood ratios varied between the three examiners. In particular, one examiner had a notably different positive likelihood ratio (3.08) compared to the other two examiners (1.22 and 1.25). It is not clear to us why this difference occurred, other than perhaps examiner 1 may have been more diligent during their clinical practice sessions. These likelihood ratios suggest, at least for two examiners, the velocimeter examination added little to their ability to predict the presence of altered blood flow (Bogduk 1999). Examiner 1 may have obtained some limited clinical advantage in detecting altered blood flow in the VA, as positive likelihood ratios of greater than 3 are considered clinically useful (Bogduk 1999; Deeks \& Altman 2004; Herbert 2004; Perera \& Heneghan 2006). 
Our study findings are in contrast to the previous study by Haynes (2000) which reported that sensitivity and specificity of using the velocimeter to detect 'marked' VA blood flow changes, confirmed by duplex ultrasound examination, were both 1 (i.e. 100\% agreement). In contrast, our study found that the velocimeter has a poor sensitivity despite $(\geq 50 \%)$ changes in PS velocity in the VA. Interestingly, Haynes (2000) reported persons with changes in PS velocity of as little as $35 \%$ had been identified by 'marked' or 'complete' loss in velocimeter (audio) signal. In our study, participants had to have a change in PS velocity of $\geq 50 \%$. In contrast to Haynes (2000), our study did not identify any participants with complete cessation of Doppler signal. However, our duplex examination did find $29 \%$ of the participants $(23.28 \%$ of arteries) had altered VA blood flow, based on our criteria (see Table 2), during contralateral rotation. Haynes (2000) found 20\% (12.5\% of arteries) of participants in his study had altered VA blood flow. Haynes' pre-selection of patients by velocimeter may have contributed to this discrepancy. Perhaps the difference in velocimeter findings between the two studies may be at least partially attributable to an examiner who had substantial experience (three years) in VA velocimeter examination.

Haynes et al (2000) undertook a further study assessing inter-examiner reliability of the velocimeter involving the experienced examiner and a trained novice. The study reported 'excellent' $(\kappa=0.78)$ agreement between examiners. This is in marked contrast to the 'poor' $(\kappa=$ 0.15 to 0.26 ) agreement among examiners in our study. We are at a loss to explain this large discrepancy between the two studies. It is possible that the training and experience of the examiners using the velocimeter in our study was inadequate in spite of including a formal training period and assessment. Our data suggest, in agreement with Haynes et al (2000), that the velocimeter is highly operator dependent. This is unacceptable for any diagnostic or screening test.

Current pre-manipulative testing procedures lack objective information on blood flow status (Refshauge 1994). The inclusion of a Doppler (velocimeter) examination of the VA as part of a pre-manipulative testing regimen is not an unreasonable proposal given the findings of Haynes (Haynes 2000). Despite our results, it could be argued that the use of the velocimeter may still 
allow detection of some individuals with abnormal VA blood flow (Thomas et al. 2007). Notably, our study suggests that the velocimeter would have identified approximately $30 \%$ more patients with altered VA blood flow than would have been detected by positional tests described in premanipulative guidelines (International Federation of Manual Medicine 1979; George et al. 1981; Rivett et al. 2006). Moreover, use of the velocimeter may assist in earlier referral of patients with potential VA blood flow alterations to a medical specialist for more comprehensive examination.

It has been considered by some authors (Haynes 2002c; Rivett et al. 2003) that marked reduction or loss of VA blood flow on neck rotation can be an indication of biomechanical stress of the VA induced by this position, and should be considered an independent risk factor for stroke (Weintraub \& Khoury 2004). It is proposed that this should be considered a contraindication to neck manipulation as a manipulative thrust might cause further stress of the artery resulting in mechanical damage to the vessel (Haynes 2000).

Importantly, it remains to be demonstrated that alterations in blood flow correlate with higher risk of cerebrovascular compromise following neck manipulation. Given that the individuals did not demonstrate any symptoms or signs of VBI it would seem that alterations in blood flow may in fact represent normal physiological variation and that the collateral flow was adequate to maintain flow to the brain.

However, a prudent clinician should attempt to gain as much clinical data about the integrity of the cerebrovascular system, especially blood flow to the brain, before considering the use of neck manipulation (Kerry \& Taylor 2006). Based on our data however, we question the validity of findings obtained from VA velocimeter examination.

In conclusion, the use of the velocimeter to detect abnormalities in VA blood flow in the upper cervical spine in neutral and end-range contralateral rotation positions is not supported by the present study. Therefore, its use as a pre-manipulative screening tool cannot be recommended at this time. Further research using a study sample with more pronounced VA blood flow 
abnormalities, such as total occlusion or cessation of flow in rotation, might yield more positive results and better define the circumstances in which examination with a Doppler velocimeter may reliably assist the clinician to determine the integrity of flow in the VA.

Acknowledgements.

We are grateful to Greg O'Connor from the Department of Diagnostic Imaging, John Hunter Hospital, NSW Australia for performing the Duplex ultrasound scans. 


\section{$\underline{\text { References }}$}

Bogduk, N. (1999). "Truth in Musculo-skeletal medicine. Truth in Diagnosis - Validity." Australasian Musculo-Skeletal Medicine May: 32-39.

Capuano-Pucci, D., Rheault, W., Aukai, J., Bracke, M., Day, R. ,Pastrick, M. (1991). "Intratester and intertester reliability of the cervical range of motion device." Archives of Physical Medicine and Rehabilitation. 72: 338-340.

Cassidy, D., Boyle, E., Cote, P., He, Y., Hogg-Johnson, S., Silver, F., L ,Bondy, S., J (2008). "Risk of Vertebrobasilar Stroke and Chiropractic Care. Results of a population-based case-control and case-crossover study." Spine 33(4S): S176-S183.

Deeks, J., J ,Altman, D., G (2004). "Diagnostic tests 4: likelihood ratios." British Medical Journal 329(July): 168-9.

Fleiss, J. L. (1971). "Measuring nominal scale agreement among many raters." Psychological Bulletin 76(5): 378-382.

Fleiss, J. L. (1986). The design and analysis of clinical experiments. New York, John Wiley \& Sons.

Frisoni, G. ,Anzola, G. (1991). "Vertebrobasilar ischaemia after neck motion." Stroke 22: 1452-60.

George, P. E., Silverstein, H. T., Wallace, H. ,Marshall, M. (1981). "Identification of the high risk pre-stroke patient." Journal of Chiropractic (Suppl): 26-28.

Haynes, M. (2000). "Vertebral Arteries And Neck Rotation: Doppler velocimeter and Duplex results compared." Ultrasound in Medicine and Biology 26(1): 57-62.

Haynes, M. (2002c). "Vertebral arteries and cervical movement: Doppler ultrasound velocimetry for screening before manipulation." Journal of Manipulative and Physiological Therapeutics 25(9): 556-567. 
Haynes, M., Hart, R. ,McGeachie, J. (2000). "Vertebral Arteries And Neck Rotation: Doppler Velocimeter Interexaminer Reliability." Ultrasound in Medicine and Biology 26(8): 13631367.

Heart Foundation (2004). Hypertension guide for doctors, www.heart foundation.org.au.

Herbert, R. (2004). The accuracy of diagnostic tests. Musculoskeletal Physiotherapy. Clinical Science and Evidence-Based Practice. K. M. Refshauge and E. Gass. Oxford, Butterworth- Heinemann: 109-116.

International Federation of Manual Medicine (1979). "On the prevention of accidents arising from manipulative therapy of the cervical spine." Declaration of the presidium of the German Association of Manual Medicine.

Kerry, R. ,Taylor, A. (2006). "Cervical arterial dysfunction assessment and manual therapy." Manual Therapy 11: 243-253.

Lee, K., Carlini, W. G., McCormick, G. F. ,Albers, G. W. (1995). "Neurologic complications following chiropractic manipulation: A survey of California neurologists." Neurology 45(June): 1213-1215.

Magarey, M., Coughlan, B. ,Rebbeck, T. (2000). "Clinical Guidelines for Pre-manipulative Procedures for the Cervical Spine." Australian Physiotherapy Association \& Musculoskeletal Physiotherapy Australia: 1-7.

Magarey, M., Rebbeck, T., Coughlan, B., Grimmer, K., Rivett, D. ,Refshauge, K. M. (2004). "Premanipulative testing of the cervical spine review, revision and new clinical guidelines." Manual Therapy 9: 95-108.

NHMRC, N. H. a. M. R. C. (2007). National Statement on Ethical Conduct in Research involving Humans. A. Government, www.nhmrc.gov.au/publications/synopses/e35syn.htm.

Perera, R. ,Heneghan, C. (2006). "Making sense of diagnostic tests likelihood ratios." Evidence Based Medicine 11(Oct): 130-131. 
Refshauge, K. M. (1994). "Rotation: A valid premanipulative dizziness test? Does it predict safe manipulation?" Journal of Manipulative and Physiological Therapeutics 17(1): 15-19.

Rheault, W., Albright, B., Byers, C., Franta, M., Johnson, A., Skowronek, M. ,Dougherty, J. (1992). "Intertester reliability of the cervical range of motion device." The Journal of Orthopaedic and Sports Physical Therapy 15: 147-150.

Rivett, D. (2001). "A valid pre-manipulative screening tool is needed." Australian Jourrnal of Physiotherapy 47: 165.

Rivett, D., Sharples, K. ,Milburn, P. (2003). "Reliability of ultrasonographic measurement of vertebral artery blood flow." New Zealand Journal of Physiotherapy 31(3): 119-128.

Rivett, D., Shirley, D., Magarey, M. ,Refshauge, K. M. (2006). APA Clinical Guidelines for Assessing Vertebrobasilar Insufficiency in the Management of Cervical Spine Disorders 2006 -. Melbourne, Australian Physiotherapy Association, Muculo-skeletal Physiotherapy Australia.

Shrout, P., E ,Fleiss, J. L. (1979). "Intraclass correlations: Uses in assessing rater reliability." Psychological Bulletin 86(2): 420-428.

Thiel, H., Bolton, J., E, Docherty, S. ,Portlock, J., C (2007). "Safety of Chiropractic Manipulation of the Cervical Spine. A Prospective National Survey." Spine 32(21): 2375-2378.

Thomas, L., Rivett, D. ,Bolton, P. S. (2007). "Comments in response to letters to editor regarding article: Thomas LC, et al Pre-manipulative testing and the velocimeter. Manual Therapy (2007)." Manual Therapy doi:10.1016/j.math.2007.09.010.

Thomas, L., Rivett, D. ,Bolton, P. S. (2008). "Pre-manipulative testing and the use of the velocimeter." Manual Therapy 13(1): 29-36.

Weintraub, M. L. ,Khoury, A. (2004). "Mechanical compression of the extracranial vertebral artery during neck rotation." Neurology 62(June): 2143-2144. 
Youdas, J. W., Carey, J. R. ,Garrett, T. R. (1991). "Reliability of measurements of cervical spine range of motion - comparison of three methods." Physical Therapy 71: 98-106.

Zwiebel, W. J. (2000). Introduction to Vascular Ultrasonography. Philadelphia, Pensylvania, Harcourt Health Sciences. 


\section{Captions for tables and figures}

Table 1 A list of findings on velocimeter examination considered to represent aberrant ('abnormal') vertebral artery blood flow.

Table 2 A list of findings on duplex examination considered to represent aberrant ('abnormal') vertebral artery blood flow.

Table 3 A table showing Intraclass correlation coefficients (ICC 2,1) and 95\% confidence intervals $(\mathrm{Cl})$ of the two duplex ultrasonographic measurements of peak systolic and end diastolic blood flow velocities in the neutral (Neutral position) and the end-range rotated neck positions (Rotation position), for the left and right vertebral arteries (VA) of each subject $(n=58)$.

Table 4 Table showing the number and distribution of vertebral arteries categorised as having, each of, aberrant peak systolic (PS) and end diastolic (ED) velocity in the left (LVA) and right (RVA) vertebral arteries in the neutral and contralateral rotation positions $(n=27)$.

Table 5 A table showing combined $2 \times 2$ contingency tables of the individual results of the comparison between duplex and velocimeter examination findings for each of the physiotherapist examiners. +ve refers to 'abnormal' vertebral artery flow.

Table 6 A table of the sensitivity, specificity and likelihood ratios for velocimeter examination for each of the individual physiotherapist examiners compared to the duplex examination findings.

Figure 1 Photograph of the Doppler traces of two vertebral arteries demonstrating i). $\geq 50 \%$ increase in peak systolic velocity from the neutral position (panel A) to the 
contralateral rotation position (panel B) and ii).loss of end diastolic flow from the neutral position (panel C) to the contralateral rotation position (panel D).

Figure 2 Graph showing the distribution of peak systolic velocity (PS) changes from the neutral position to the contralateral neck rotation position for left (LVA) and right (RVA) vertebral arteries. 
Table 1

- absence of signal in neutral

- cessation of signal in contralateral rotation

- marked decrease in signal in contralateral rotation

- marked increase in signal in contralateral rotation. 
Table 2

- absence of peak systolic flow in neutral or contralateral rotation positions

- absence of end diastolic flow in neutral or contralateral rotation positions

- $50 \%$ or more change in peak systolic flow velocity between neutral and rotation positions 
Table 3

\begin{tabular}{|l|c|c|}
\hline \multicolumn{1}{|c|}{ Measure } & Neutral position & Rotation position \\
& ICC (95\% CI) & ICC (95\% CI) \\
\hline Left VA peak systolic & $0.71(0.56-0.82)$ & $0.72(0.56-0.83)$ \\
Left VA end diastolic & $0.83(0.73-0.89)$ & $0.72(0.56-0.82)$ \\
Right VA peak systolic & $0.79(0.67-0.87)$ & $0.85(0.75-0.97)$ \\
Right VA end diastolic & $0.79(0.67-0.87)$ & $0.77(0.65-0.86)$ \\
\hline
\end{tabular}


Table 4

\begin{tabular}{|c|c|c|c|}
\hline & L VA & R VA & Total \\
\hline Absent PS flow neutral & 0 & 0 & 0 \\
Cessation PS flow rotation & 0 & 0 & 0 \\
$\geq 50 \%$ increase PS velocity & 1 & 4 & 5 \\
$\geq 50 \%$ decrease PS velocity & 1 & 0 & 3 \\
\hline Absent ED flow neutral & 3 & 0 & 6 \\
Cessation ED flow rotation & 3 & 3 & 27 \\
Absent ED flow throughout & 6 & 6 & \\
\hline Total & 14 & & \\
\hline
\end{tabular}


Table 5

\begin{tabular}{|c|c|c|c|c|c|c|c|c|c|c|}
\hline & \multicolumn{4}{|c|}{ Examiner 1} & \multicolumn{3}{|c|}{ Examiner 2} & \multicolumn{3}{|c|}{ Examiner 3} \\
\hline & \multicolumn{3}{|c|}{ Duplex } & \multirow[t]{2}{*}{ Total } & \multicolumn{2}{|c|}{ Duplex } & \multirow[t]{2}{*}{ Total } & \multicolumn{2}{|c|}{ Duplex } & \multirow[t]{2}{*}{ Total } \\
\hline & & +ve & -ve & & +ve & -ve & & +ve & -ve & \\
\hline & +ve & 6 & 5 & 11 & 4 & 9 & 13 & 4 & 8 & 12 \\
\hline Velocimeter & -ve & 10 & 36 & 46 & 11 & 32 & 43 & 12 & 33 & 45 \\
\hline Total & & 16 & 41 & 57 & 15 & 41 & 56 & 16 & 41 & 57 \\
\hline
\end{tabular}


Table 6

\begin{tabular}{|l|c|c|c|}
\hline & Examiner 1 & Examiner 2 & Examiner 3 \\
\hline Sensitivity & 0.38 & 0.27 & 0.25 \\
\hline Specificity & 0.88 & 0.78 & 0.80 \\
\hline +ve likelihood ratio & 3.08 & 1.22 & 1.25 \\
\hline & & & 0.94 \\
\hline -ve likelihood ratio & 0.72 & 0.94 & \\
\hline
\end{tabular}



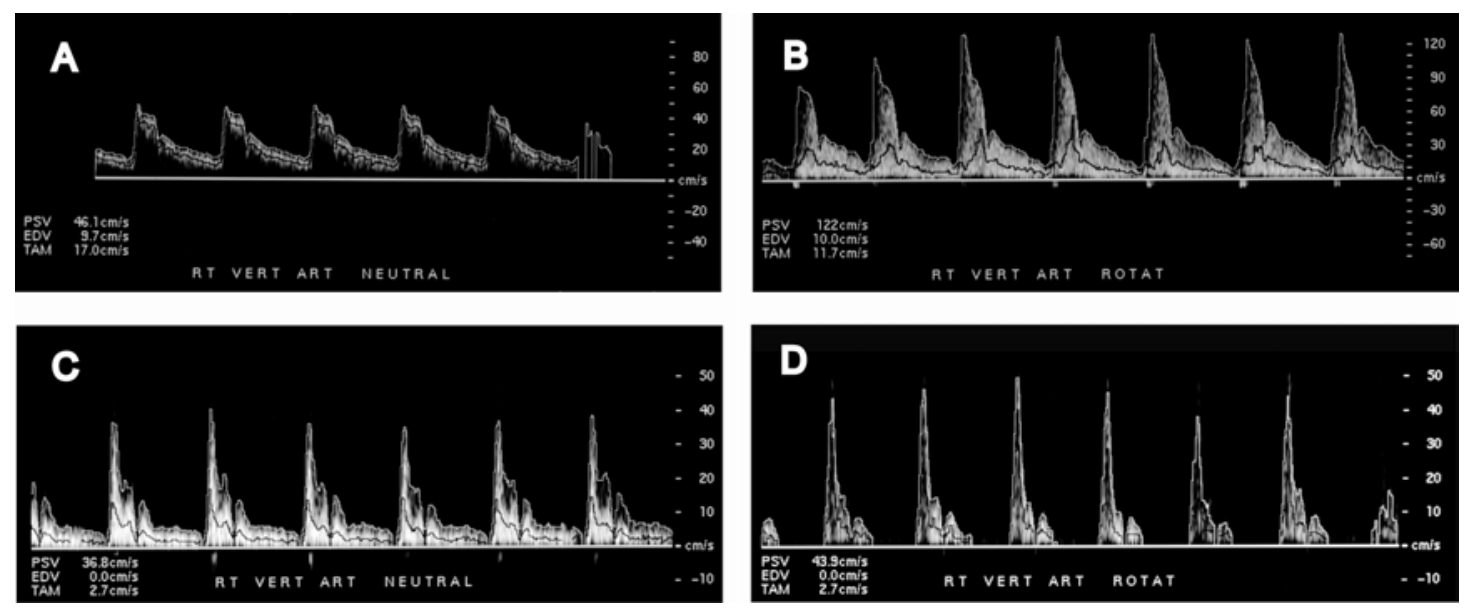
Figure 2

Distribution of Peak Systolic Changes on Rotation

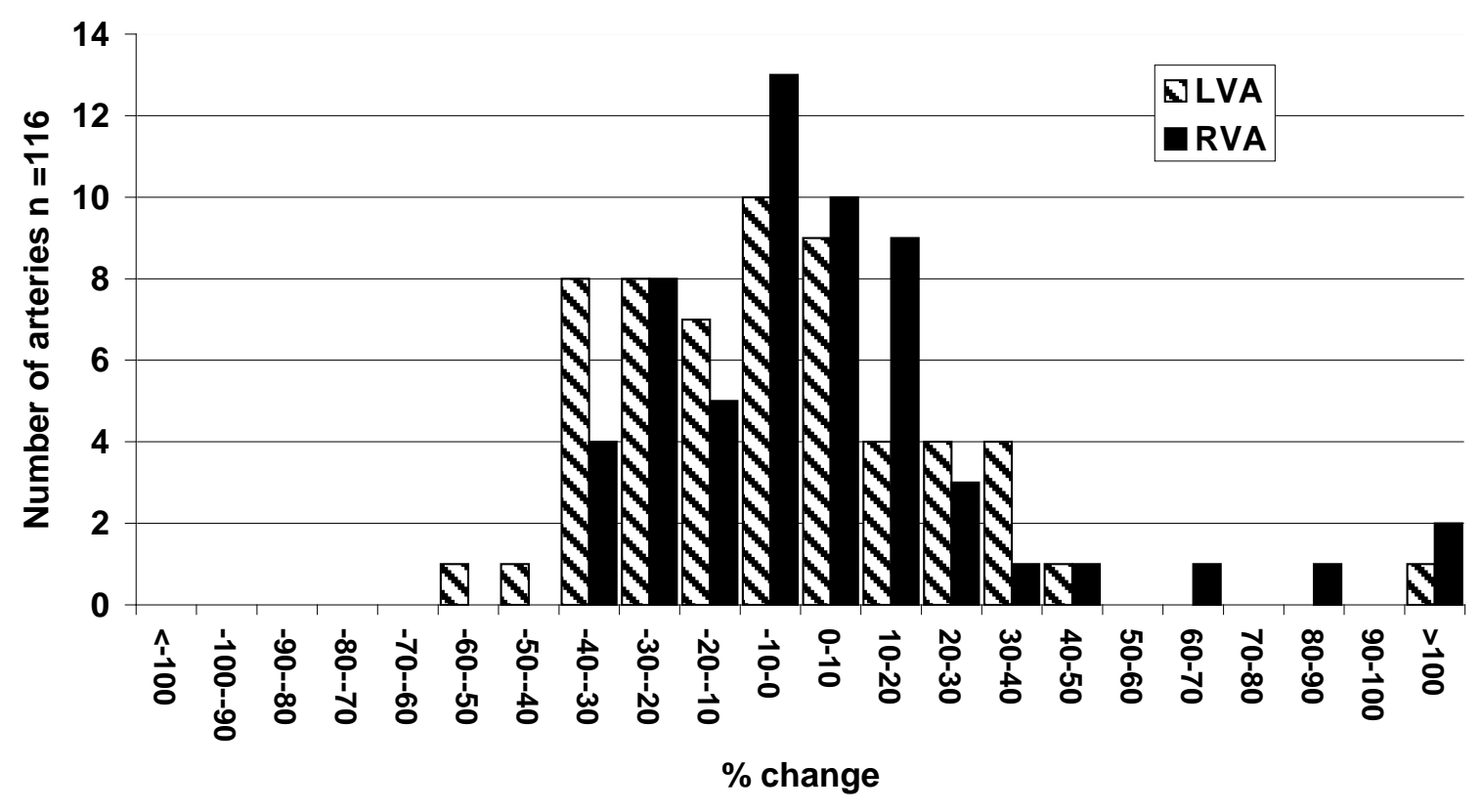

